\title{
Banana quality attribute prediction and ripeness classification using support vector machine
}

\begin{abstract}
Five laser diodes of 532, 660, 785, 830 and 1060nm laser light back scattering imaging (LLBI) were employed for quality attribute prediction and ripening stage classification of banana. A support vector machine (SVM) was tested to establish the theoretical prediction and classification models to predict chlorophyll, elasticity and soluble solids content (SSC) and also to classify the bananas into six ripening stages. The classification was set up with six ripening stages 2-7. Wavelengths of 532, 660 and 785nm gave high correlation coefficients both for banana quality prediction and ripeness classification. The results show that the highest correlation coefficients of $0.912,0.945$ and 0.872 were obtained for chlorophyll, elasticity and SSC at 785, 660nm respectively. An overall classification accuracy of $92.5 \%$ was recorded at $830 \mathrm{~nm}$. These results show that LLBI with the SVM model can be used for non-destructive estimation of banana quality attributes and the subsequent ripeness classification.
\end{abstract}

Keyword: Laser diodes; Banana; Elasticity; Ripeness; Chlorophyll; Quality 